\author{
Peter J. Strouse \\ Frank Londy \\ Michael A. DiPietro \\ Eu-Leong H.J. Teo \\ Clarence E. Chrisp \\ Kei Doi
}

\section{MRI evaluation of infectious and non-infectious synovitis: preliminary studies in a rabbit model}

Received: 8 May 1998

Accepted: 27 October 1998

This project was supported by the Bracco Society for Pediatric Radiology Research Grant

P.J.Strouse ( $)$ M. A. DiPietro ·

E.-L.H.J.Teo

Section of Pediatric Radiology/F3503,

C.S. Mott Children's Hospital,

University of Michigan Health System,

1500 E. Medical Center Drive, Ann Arbor,

MI 48109-0252, USA

\section{F. Londy}

Division of Magnetic Resonance Imaging,

Department of Radiology,

University of Michigan Health System,

1500 East Medical Center Drive,

Ann Arbor, MI 48109-0252, USA

\section{E. Chrisp}

Unit of Laboratory Animal Medicine,

018 Animal Research Facility,

University of Michigan Health System,

Ann Arbor, MI 48109-0614, USA

\section{K. Doi}

Division of Musculoskeletal Radiology, Department of Radiology,

University of Michigan Health System,

1500 East Medical Center Drive,

Ann Arbor, MI 48109-0252, USA
Abstract Background. Literature on magnetic resonance imaging (MR) evaluation of inflammatory joint effusions is sparse.

Objective. To describe an animal

model for studying infectious and non-infectious joint effusions with magnetic resonance imaging. Materials and methods. Ten rabbit knees with septic arthritis and four with talc synovitis were imaged with MR. Contralateral knees injected with saline served as controls. Fat saturation T2-weighted and gadolinium-enhanced T1-weighted images were assessed for joint effusion, and periarticular and adjacent intraosseous increased signal or enhancement. Each knee was cultured and underwent pathologic examination. Results. Both Staphylococcus aureus and talc produced effusions in all knees. The degree of periarticular signal and enhancement was greater in infected knees than talc-injected knees. No abnormal enhancement was seen within bone. Pathologic examination showed a greater degree of inflammation and joint destruction in the infected knees, but no evidence of osteomyelitis.

Conclusion. A greater degree of ab- normal signal and enhancement seen on MR suggests a more vigorous inflammatory process, as seen with septic arthritis. In spite of advanced septic arthritis, no enhancement was evident within bone, suggesting that enhancement within bone is not an expected finding in isolated septic arthritis and should raise concern for osteomyelitis.

\section{Introduction}

Septic arthritis has the potential to produce substantial morbidity and permanent joint deformity [1]. It may present in association with or lead to osteomyelitis with- in the adjacent bone [2-4]. Although magnetic resonance imaging (MR) has been used extensively to study inflammatory conditions, notably osteomyelitis, there is relatively little information on the utility of MR in the evaluation of suspected septic arthritis [2-9]. 
In this preliminary investigation, we have used MR to study rabbit knees with experimentally produced septic arthritis and talc synovitis in comparison to control knees injected with saline. Our hypothesis in undertaking this study was that greater degrees of abnormality are seen with infectious synovitis than non-infectious synovitis and that MR can accurately assess the extension of infection into adjacent structures, including bone.

\section{Materials and methods}

\section{Rabbit model}

The research protocol was approved by the University Committee on Use and Care of Animals. Three-kilogram adult white New Zealand rabbits were utilized. For injection and imaging the rabbits were anesthetized with ketamine $(35 \mathrm{mg} / \mathrm{kg})$ and xylazine $(5 \mathrm{mg} /$ $\mathrm{kg}$ ) intramuscularly. This provided approximately 30-45 min of sedation, sufficient time for our MR imaging protocol in all rabbits.

\section{Injection}

The rabbit knees were aseptically prepared with tincture of iodine. The injection site was identified by palpating the patella and the proximal margin of the tibia. A needle was passed through patellar tendon and into the joint (approximately $1 \mathrm{~cm}$ depth). On proper placement of the needle into the joint, injection was easy and without resistance. Injections of saline and Staphylococcus aureus suspensions were performed with a 25 -gauge needle whereas injection of talc suspension required a 18-gauge needle.

Ten rabbit right knees were injected with 1,000 colony-forming units of $S$. aureus suspended in $1 \mathrm{ml}$ of sterile saline. We used ATCC 27217 strain of S. aureus (American Type Culture Collection, Rockville, Md.). This strain has been shown to achieve a high rate of septic arthritis in the rabbit [10]. Four rabbit right knees were injected with $0.125 \mathrm{~g}$ of sterile surgical talc in $1 \mathrm{ml}$ of sterile saline. This model was adapted from previous studies of talc synovitis in the rabbit $[11,12]$. The left knees of each of the aforementioned 14 rabbits were injected with $1 \mathrm{ml}$ of sterile saline to serve as internal controls.

\section{Imaging}

Rabbits with septic arthritis were imaged 3 or 4 days after injection when a septic arthritis was clinically evident. Rabbits with talc synovitis were imaged 14 days after injection when a synovitis was clinically evident. The timing of imaging was based on our clinical and sonographic observation of the rabbits and descriptions of the course of inflammation in the literature [10-14]. We attempted to image at a time when the inflammatory response would be fully established. Imaging was performed on a 1.5-Tesla scanner (GE Medical Systems, Milwaukee, Wis.). Each sedated rabbit was placed supine with its knees centered in an extremity coil. The knees were taped together and padding was placed in the coil to maintain positioning and limit the field of view and number of slices necessary. A coronal fast spin echo T1-weighted sequence was obtained first for localization $(\mathrm{TR}=650 \mathrm{~ms}$, TE $=12 \mathrm{msec}$, $10 \mathrm{~mm}$ thick skipping $2 \mathrm{~mm}, 12 \mathrm{~cm}$ field of view, $256 \times 128$ matrix, 1 excitation). Subsequently, a sagittal fast spin echo T2-weighted with fat saturation sequence was obtained $(T R=4000 \mathrm{~ms}$,
$\mathrm{TE}=85 \mathrm{~ms}, 3 \mathrm{~mm}$ thick skipping $2 \mathrm{~mm}, 12 \mathrm{~cm}$ field of view, $256 \times 256$ matrix, 2 excitations). Finally, sagittal spin echo T1weighted with fat saturation images were obtained before and after the administration of gadolinium (TR $=650 \mathrm{~ms}, \mathrm{TE}=12 \mathrm{~ms}, 3 \mathrm{~mm}$ thick skipping $2 \mathrm{~mm}, 12 \mathrm{~cm}$ field of view, $256 \times 256$ matrix, 2 excitations). The rabbits received $1 \mathrm{mmol} / \mathrm{kg}$ of gadolinium (Omniscan, Nycomed, Princeton, N.J.) injected via a 25 -gauge butterfly needle into a marginal ear vein.

\section{Laboratory analysis}

The rabbits were euthanized under protocol approved and recommended by the University Committee on Use and Care of Animals. Each rabbit knee was aspirated for culture. When no fluid was obtained, $1 \mathrm{ml}$ of sterile saline was injected, aspirated, and submitted for culture. Each rabbit knee underwent pathologic examination. After dissection and decalcification, hematoxylin and eosin (H \& E) stains were performed. The degree of inflammatory changes histologically present was assessed. Specific evidence of osteomyelitis was sought.

Data analysis

Due to artifact produced by talc crystals, it was not possible to assess the scans with truly blinded observers. Images were assessed by the authors for the presence of effusion, periarticular soft tissue T2 signal abnormality and gadolinium enhancement, and adjacent intraosseous T2 signal abnormality and gadolinium enhancement.

\section{Results}

Effusions were present within all S. aureus injected knees and all talc-injected knees. The fluid was predominantly posterior within the knee joint, but also within the suprapatellar pouch. A tiny amount of fluid was present within the saline-injected control knees, presumably within physiologic limits. Artifact was produced by the injected talc. The degree of increased periarticular signal on T2-weighted images and the degree of enhancement after gadolinium administration on the T1-weighted images were greater in the infected knees than the talcinjected knees (Figs. 1, 2). The periarticular abnormality was diffuse, much broader than could be ascribed to direct inoculation. The control knees showed no definite increased periarticular signal or enhancement. No definite intraosseous increased signal or enhancement was noted in either infected or talc-injected knees in comparison to the control knees. All knees showed mild increased T2 signal and slight enhancement within the metaphyses, likely reflecting some residual hematopoetic marrow in this region (confirmed histologically).

Cultures confirmed infection in all of the S. aureus injected knees, but did not show infection in any talc or saline injected knee. On pathologic examination, no abnormality was evident in the control knees (Fig. $3 \mathrm{a}$ ). Talc-injected knees showed a mononuclear cellular infiltrate, with an intact synovium (Fig. $3 \mathrm{~b}$ ). The infected 

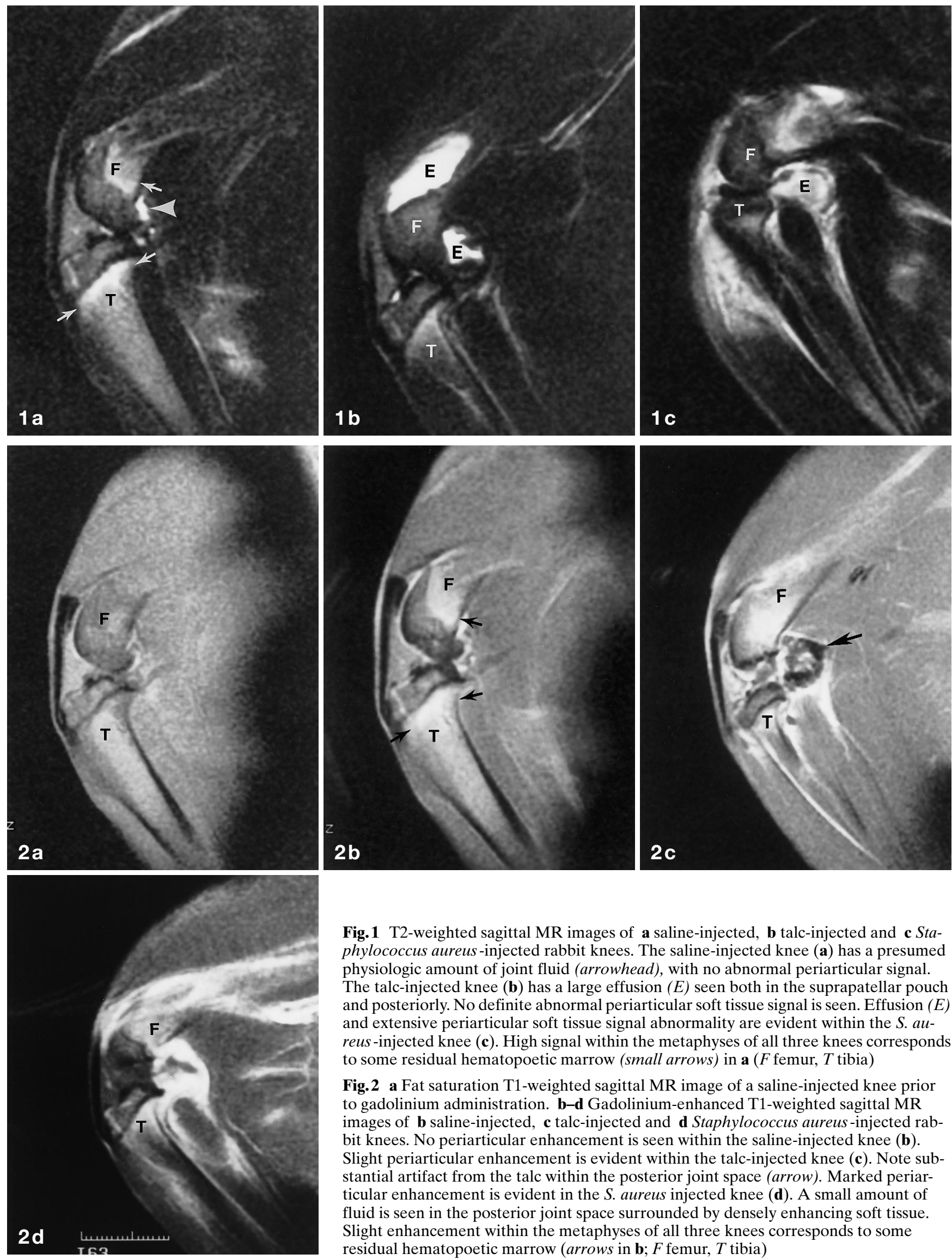

Fig. 1 T2-weighted sagittal MR images of a saline-injected, b talc-injected and c Staphylococcus aureus -injected rabbit knees. The saline-injected knee (a) has a presumed physiologic amount of joint fluid (arrowhead), with no abnormal periarticular signal. The talc-injected knee (b) has a large effusion $(E)$ seen both in the suprapatellar pouch and posteriorly. No definite abnormal periarticular soft tissue signal is seen. Effusion $(E)$ and extensive periarticular soft tissue signal abnormality are evident within the $S$. aureus-injected knee (c). High signal within the metaphyses of all three knees corresponds to some residual hematopoetic marrow (small arrows) in a ( $F$ femur, $T$ tibia)

Fig. 2 a Fat saturation T1-weighted sagittal MR image of a saline-injected knee prior to gadolinium administration. b-d Gadolinium-enhanced T1-weighted sagittal MR images of b saline-injected, c talc-injected and d Staphylococcus aureus-injected rabbit knees. No periarticular enhancement is seen within the saline-injected knee (b). Slight periarticular enhancement is evident within the talc-injected knee (c). Note substantial artifact from the talc within the posterior joint space (arrow). Marked periarticular enhancement is evident in the $S$. aureus injected knee (d). A small amount of fluid is seen in the posterior joint space surrounded by densely enhancing soft tissue. Slight enhancement within the metaphyses of all three knees corresponds to some residual hematopoetic marrow (arrows in $\mathbf{b} ; F$ femur, $T$ tibia) 

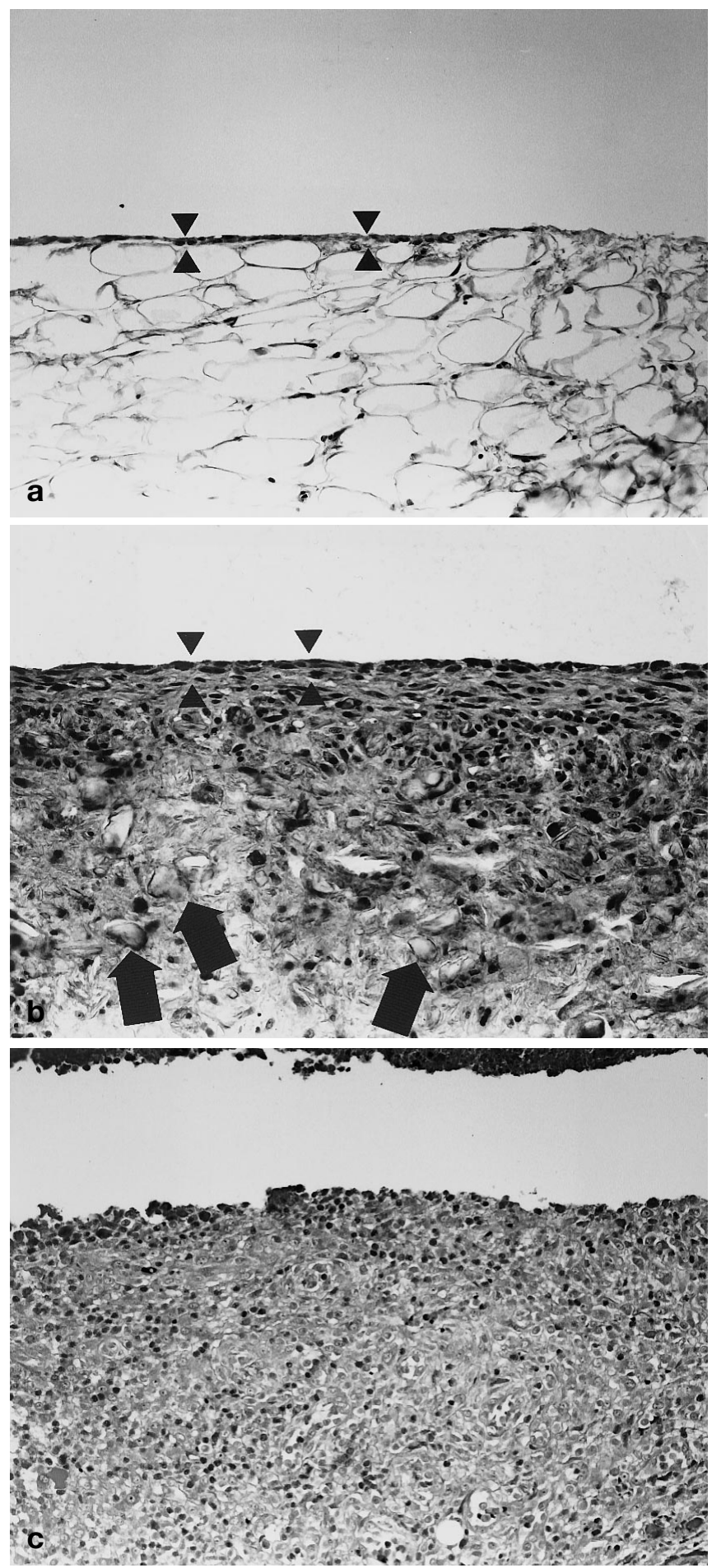

Fig.3 Histologic examination of a saline-injected, b talc-injected and c Staphylococcus aureus injected rabbit knees. The saline-injected control (a) shows normal, thin synovial lining (arrowheads). The talc-injected knee (b) shows a mononuclear cell infiltrate, but an intact synovial membrane (arrowheads). Note talc crystals (arrows). The $S$. aureus injected knee (c) shows an even greater degree of cellular infiltrate with neutrophils. The synovial membrane is destroyed and difficult to identify knees showed a marked polymorphonuclear cell infiltrate, with frank destruction of the synovium (Fig. $3 \mathrm{c}$ ). In some rabbits there was evidence of extension of the infectious process into the adjacent soft tissues. On sections obtained, there was no evidence of osteomyelitis in any knee.

\section{Discussion}

We have presented our preliminary observations on the study of joint effusions with MR using an animal model. Based on the culture and pathological results obtained, the model was successful in producing septic arthritis and a non-infectious synovitis and can be used for further investigations. The results of our study show that septic arthritis produces a greater degree of periarticular signal abnormality and enhancement than talc synovitis, reflecting a more vigorous inflammatory response seen on pathologic examination.

The applicability of our results to the human population is limited. While talc synovitis is a non-infectious synovitis like the transient synovitis seen in children, its etiology and pathophysiology are defined, whereas the etiology and pathophysiology of transient synovitis are unknown. Thus, there is no ideal animal model for transient synovitis. We used talc to produce synovitis because its efficacy in our chosen rabbit model had been previously described [11,12]. Gershuni and Kuei postulated that the non-specific inflammatory effects of talc synovitis are similar to those of transient synovitis; however, this remains unproven [12]. The talc synovitis model has not been previously studied by MRI.

In our study, no signal abnormality or enhancement was evident within the adjacent osseous structures, and pathologic examination showed no evidence of osteomyelitis. As the rabbits were imaged with MR well into their course of septic arthritis, and joints showed extensive synovial destructive changes, this observation suggests that associated intraosseous signal abnormality and enhancement are not a normal expected finding with septic arthritis and therefore should raise concern for osteomyelitis. Other studies, however, have suggested that periarticular bone enhancement can occur without osteomyelitis in the setting of septic arthritis $[4,9]$. Pathologic proof is limited. Our own study is limited by the lack of bone cultures confirming the absence of osteomyelitis, although neither MR images or the pathologic specimens suggested infection in the bone. Further study of adjacent bone signal characteristics and enhancement in the presence of septic arthritis is warranted.

There are other limitations to our study that should be addressed. We only assessed the MR findings at one point in time. Further study is necessary to establish the time course of MR signal abnormality and enhancement. Early in the course of septic arthritis, it is likely 
that there are lesser degrees of signal abnormality and enhancement, which could closely match that seen in established non-infectious synovitis. We evaluated the images subjectively, as we could not effectively blind observers as to the injected substance. We studied an animal model. As noted, the applicability of results to human patients may be limited. We imaged at one point in time using this controlled model, that is, rabbit knees that were infected with a known amount of known strain of bacteria. In clinical practice, numerous factors including the virulence of the infecting bacteria, the size of the inoculum and the duration since inoculation, will affect the inflammatory response and therefore the MR findings. It is likely that many patients with septic arthritis present before the inflammatory response is fully established. In such patients, the MR findings may not be as substantial as those seen in this study.
In conclusion, we have presented our preliminary observations of the MR findings of septic arthritis and a non-infectious (talc) synovitis in an animal model. Our findings suggest that a greater degree of periarticular signal abnormality and enhancement is seen in the presence of a vigorous inflammatory response such as with septic arthritis. Abnormal signal and enhancement within the adjacent bone are not expected findings even with well-established septic arthritis and should raise concern for osteomyelitis. This study and the animal model described can form the basis for future MR investigations of infectious and non-infectious synovitis.

Acknowledgements The authors thank Steven Cendroski for microbiology services, Todd Jackson, D. V.M., for assistance in preparing the animal use application, and Salo Papola for animal care.

\section{References}

1. Betz RR, Cooperman DR, Wopperer JM, et al (1990) Late sequelae of septic arthritis of the hip in infancy and childhood. J Pediatr Orthop 10: 365-372

2. Jaramillo D, Treves ST, Kasser JR, et al (1995) Osteomyelitis and septic arthritis in children: appropriate use of imaging to guide treatment. AJR 165: 399-401

3. Lee SK, Kim Y, Suh K, et al (1997) Signal alterations in bone marrow around the hip joint on MR imaging: can differentiate septic arthritis from transient synovitis? RSNA Scientific Meeting Program, p 256 (abstract)

4. Hopkins KL, Li KCP, Bergman G (1995) Gadolinium-DTPA-enhanced magnetic resonance imaging of musculoskeletal infectious processes. Skeletal Radiol 24: 325-330

5. Dangman BC, Hoffer FA, Rand FF, et al (1992) Osteomyelitis in children: gadolinium-enhanced MR imaging. Radiology 182: 743-748
6. Terrier F, Revel D, Reinhold CF, et al (1986) Contrast-enhanced MRI of periarticular soft tissue changes in experimental arthritis of the rat. Magn Reson Med 3: 385-396

7. Whitten CG, Moore TE, Yuh WTC, et al (1992) The use of intravenous gadopentetate dimeglumine in magnetic resonance imaging of synovial lesions. Skeletal Radiol 21: 215-218

8. Ranner G, Ebner F, Fotter R, et al (1989) Magnetic resonance imaging in children with acute hip pain. Pediatr Radiol 20: 67-71

9. Erdman WA, Tamburro F, Jayson HT, et al (1991) Osteomyelitis: characteristics and pitfalls of diagnosis with MR imaging. Radiology 180: 533-539

10. Daniel D, Akeson W, Amiel D, et al (1976) Lavage of septic joints in rabbits: effect of chondrolysis. J Bone Joint Surg [Am] 58: 393-395
11. Gershuni-Gordon DH, Axer A (1974) Synovitis of the hip joint: an experimental model in rabbits. J Bone Joint Surg [Br] 56: 69-77

12. Gershuni DH, Kuei SC (1984) Articular cartilage deformation following experimental synovitis in the rabbit hip. J Orthop Res 1: 313-318

13. Riegels-Nielson P, Frimodt-Moller N, Jensen SJ (1987) Rabbit model of septic arthritis. Acta Orthop Scand 58: 14-19

14. Johnson AH, Campbell WG, Callahan BC (1970) Infection of rabbit knee joints after intra-articular injection of Staphylococcus aureus. Am J Pathol 60: 165-203

15. Herve' Somma CMP, Sebag GH, Priur A-M, et al (1992) Juvenile rheumatoid arthritis of the knee: MR evaluation with Gd-DOTA. Radiology 182: 93-98 